\title{
Ambiances
}

anbiances Environnement sensible, architecture et espace urbain Varia | 2021

\section{Une critique de l'urbain depuis le champ des ambiances}

Perspectives

A critique of the urban from the field of ambiences. Perspectives

\section{Rachel Thomas}

\section{(2) OpenEdition}

\section{Journals}

Édition électronique

URL : https://journals.openedition.org/ambiances/3805

DOI : 10.4000/ambiances.3805

ISSN : 2266-839X

Éditeur :

Direction Générale des Patrimoines - DAPA - MCC, UMR 1563 - Ambiances Architectures Urbanités (AAU)

\section{Référence électronique}

Rachel Thomas, "Une critique de l'urbain depuis le champ des ambiances », Ambiances [En ligne], Varia, mis en ligne le 23 novembre 2021, consulté le 26 novembre 2021. URL : http://

journals.openedition.org/ambiances/3805; DOI : https://doi.org/10.4000/ambiances.3805

Ce document a été généré automatiquement le 26 novembre 2021.

\section{cc) (†) $९$}

Ambiances is licensed under a Creative Commons Attribution-NonCommercial-NoDerivatives 4.0 International License. 


\title{
Une critique de l'urbain depuis le champ des ambiances
}

\author{
Perspectives
}

A critique of the urban from the field of ambiences. Perspectives

\author{
Rachel Thomas
}

\section{NOTE DE L'AUTEUR}

Cet article programmatique actualise les arguments développés dans : Thomas, Rachel. 2018. Une critique sensible de l'urbain. HDR en Sciences Humaines et Aménagement. Grenoble : Université Grenoble Alpes, Cresson.

1 La construction d'une problématique des ambiances (Augoyard, 1998; Amphoux et al., 1998 ; Thibaud, 2002; Amphoux, Chelkoff \& Thibaud, 2004) au début des années 2000 répondait à la nécessité de développer une analyse des processus à l'œuvre entre les qualités sensibles des espaces urbains et la perception humaine. Elle se détachait d'une esthétique des beaux-arts, centrée sur l'étude de la sensibilité artistique et la définition de la notion de beau. Dans une perspective première d'esthétique environnementale, les travaux mettaient en avant le potentiel configurateur de l'ambiance ainsi que son efficace dans les situations banales de la vie urbaine. Composée et changeante, produit de configurations physique et sociale toujours en mouvement, l'ambiance n'est jamais neutre. Elle offre des prises pour l'action et l'interaction, modèle les façons de percevoir et d'agir en situation. L'ambiance est là où l'on se situe. Elle constitue une «toile de fond" à partir de laquelle se déploient nos expériences (Augoyard, 2011 ; Thibaud, 2012).

2 C'est précisément en décrivant des formes d'expérience en situation que ces travaux contribuent, depuis peu, à rendre explicite la manière dont les ambiances affectent les formes sensibles de la vie sociale. Parce qu'elles confèrent une certaine tonalité aux milieux que nous habitons et aux situations dans lesquelles nous sommes et nous 
agissons, elles opèrent discrètement sur nos sensibilités, sur nos manières d'être et d'être-ensemble en public. Des travaux récents mettent en évidence les effets de certaines « politiques des ambiances » en matière de marketing urbain (Breviglieri, 2013, 2019; Thomas, 2010, 2020), de sécurisation et d'hygiénisme (Margier, 2017 ; Masson et al., 2014) ou de pacification (Thomas et al., 2014) sur l'expérience sensible ordinaire et la reconfiguration de la civilité ${ }^{1}$ en public. En transformant par petites touches souvent imperceptibles nos gestes, nos modes d'attention, voire nos « états de corps » (ibid.), les ambiances affectent nos manières de sentir et d'habiter sensiblement les milieux dans lesquels nous sommes.

3 Dans le champ voisin des atmosphères ${ }^{2}$, ce sont les propriétés atmosphériques environnant les situations qui sont plus particulièrement étudiées. Les travaux révèlent comment celles-ci donnent "un ton » plus ou moins favorable à l'engagement des individus (Adey et al., 2013 ; Edensor, 2015 ; Edensor \& Sumartojo, 2015 ; Simpson, 2014 ; Sumartojo \& Pink, 2019), voire les placent dans des « états » susceptibles de les mettre en confiance ou de les déstabiliser (Böhme, 2017a; Massumi, 2015).

4 Quelques-uns de ces travaux dépassent aujourd'hui la visée descriptive qui fut première dans le champ des ambiances pour s'inquiéter des effets à long terme de leurs transformations planifiées. Ils ouvrent ainsi un champ de réflexion fécond sur les enjeux sociétaux, voire politico-moraux, posés par ces mutations et en dessinent en creux une critique.

5 C'est à la clarification de cette forme de critique que cet article prospectif s'attèle. Il s'agit de montrer - à partir d'un état de l'art des travaux récents sur la transformation programmée des ambiances - de quelle manière le détour par les ambiances nourrit un examen raisonné de leurs effets sur les formes de la vie urbaine. En quoi une telle critique peut-elle rendre intelligibles des logiques d'emprise peu perceptibles, opérant au quotidien sur nos sensibilités et nos modalités d'existence ? À travers ces questions, cet article tente d'articuler plus étroitement le champ des ambiances avec la question du politique. Il s'agit de se demander comment transporter la critique sociale sur le terrain des ambiances. Trois postures de travail sont proposées : saisir du politique dans les ambiances; historiciser la chronicité des mutations d'ambiance ; s'engager en chair et au long cours dans l'enquête. Elles nous amènent à préciser les formes que peut prendre cette critique de l'urbain depuis le champ des ambiances et les possibles qu'elle dessine.

\section{Ambiances et vivre-ensemble. Une articulation à rendre intelligible}

\section{D'une exhortation à penser une conception ménageant les sens...}

6 La critique n'est pas absente des travaux francophones sur les ambiances architecturales et urbaines. Mais elle est implicite et exclusivement formulée à l'égard des champs de la conception et de l'aménagement urbain. Trois reproches leur sont adressés: négliger les dimensions sensibles de l'espace au profit des dimensions techniques et normatives (Augoyard, 1995; Chelkoff \& Thibaud, 1992); lorsque le sensible est pris en compte dans les projets, le réduire à la seule dimension du regard au détriment des autres sens (Augoyard, 1991); utiliser les ambiances à des fins de « plus-value » esthétique (Amphoux, Thibaud \& Chelkoff, 2004 ; Augoyard, 2000). 
7 La construction d'une théorie des ambiances architecturales et urbaines accompagne, à ses débuts, un mouvement de dénonciation des pratiques de maîtrise des ambiances dans le champ de la conception. Elle fait valoir la nécessité d'une prise en compte des pratiques sociales et de leur dimension sensible :

La recherche sur les ambiances in situ engage une reprise fondamentale du caractère naturellement interdisciplinaire de la théorie et de la pratique architecturales, c'est-à-dire la reconnaissance des interdépendances entre forme construite, forme perçue, forme représentée. Impossible d'expliquer le fond de l'une de ces modalités sans se référer aux autres. (Augoyard, 1998, p. 22)

Des travaux sur la conception sonore et le vécu des espaces hospitaliers (Leroux et Bardyn, 2003), sur les formes sensibles de l'architecture (Chelkoff, 2005) ou encore sur la monumentalisation des espaces publics (Juan Cantavella, 2009) exhortent les milieux de l'architecture et de l'aménagement urbain à penser une conception ménageant les sens.

Devenue classique dans le champ des ambiances architecturales et urbaines, cette critique pourrait être rapprochée de la Critique de la vie quotidienne d'Henri Lefebvre (1947), et plus largement des pensées critiques d'inspiration marxistes qui ont nourri la recherche urbaine depuis. Mais elle s'en démarque pour deux raisons. D'une part, elle évacue la question de la domination et des inégalités pour se focaliser sur la pluralité des modes ordinaires d'habiter. Se désintéressant des explications déterministes qui font des disparités économiques ou socio-spatiales les principales causes du malaise habitant, les travaux menés dans le champ des ambiances s'intéressent aux pratiques effectives des citadins et à l'inventivité dont ils font preuve pour se soustraire aux contraintes des territoires planifiés. D'autre part, l'approche depuis les ambiances se veut modale et non plus causale, substituant un comment au pourquoi d'Henri Lefebvre. Elle dévoile les capacités d'adaptation des citadins, leurs compétences pratiques et perceptives plutôt que de supposer - dans une vision désillusionnée de la vie - leur apathie. Des travaux empiriques révèlent, par exemple, comment des formes d'intimité s'élaborent au quotidien (par la création de bulles sonores ou visuelles, par des stratégies d'évitement visuel ou l'installation de dispositifs physiques...) dans des zones d'habitat individuel dense (à Bordeaux et Paris) où le vis-à-vis et la promiscuité rendent la construction d'un « chez-soi » difficile (Atienza et al., 2007).

10 Progressivement, les travaux de ce champ, en s'ouvrant à l'international, confrontent les chercheurs à des terrains problématiques sur le plan sensible et social. C'est le cas notamment de travaux empiriques interrogeant les épreuves de la mobilité piétonne en situation de labilité à Caracas (Fiori \& Sanchez, 2014), de sur-surveillance en public dans les gares du Nord à Paris et de Londres St Pancras (Masson et al., 2014) ou de pacification à Bahia (Thomas et al., 2014). La portée critique de ces travaux réside dans ce que j'appelle une phénoménologie de l'implicite. C'est d'une part le niveau d'orchestration programmée des usages contenu dans certaines politiques d'ambiance qui est révélé. C'est d'autre part l'opérationnalité des ambiances sur la reconfiguration de la civilité qui est éclairée.

11 Ainsi, un travail d'enquête de quatre ans à Salvador de Bahia au Brésil - dans le contexte de la préparation d'évènements sportifs mondiaux (Copa, Jeux olympiques) - a montré comment la mise en place d'une politique de pacification des espaces urbains, destinée entre autres à parfaire l'image de la ville, a progressivement bouleversé les pratiques sociales et les modalités de l'être-ensemble en public. A contrario du 
programme de pacification des favelas voté en 2008, cette politique a opéré en mode mineur, c'est-à-dire sans énonciation claire dans une stratégie légitimée par l'État, mais à travers diverses mesures de revitalisation urbaine, de sécurisation et d'assainissement des espaces publics. Embellis, nettoyés, désencombrés (le nombre des vendeurs ambulants a été considérablement réduit au fil des ans) et sécurisés (par la présence constante d'une police militaire armée et mobile), Praça da Piedade et Porto da Barra, les deux quartiers dans lesquels nous avons enquêté, ont ainsi vu les enfants des rues disparaitre, les touristes passer plutôt que séjourner, les pratiques habitantes moralisées par la multiplication de campagnes de sensibilisation à la salubrité publique, les rassemblements spatialement encadrés par des dispositifs aménagés (grille, fermeture des rues, barrières, etc.). Sous couvert de ramener l'ordre, le calme et le propre là où le désordre, l'agitation et le sale régnaient, ces mesures de pacification ont affecté les ambiances ordinaires des quartiers. Elles ont installé, selon les moments, un climat de tension, de malaise ou d'apathie dont nous avons observé les effets sur les manières de se côtoyer (plus distanciées et moins spontanées), d'appréhender l'Autre (sous le régime de la suspicion plutôt que de l'indifférence polie), de se sentir soi-même vulnérable, en tension (Thomas et al., 2014).

Timide, une nouvelle piste critique s'ouvre ici, qui interroge explicitement le devenir des formes sensibles de la vie urbaine et des sensibilités.

\section{... à la clarification des enjeux de la transformation programmée des ambiances urbaines}

13 C'est également à ces questions que s'attèlent divers travaux sur la fabrique des ambiances de la ville néolibérale. L'objet de leur critique est double : la production de décors standardisés, soutenue par une attention accrue à l'esthétisme des aménagements, conduirait non seulement à une aseptisation des ambiances urbaines, mais aussi à une remise en question des qualités d'hospitalité de la ville.

Marc Breviglieri (2013) pose les termes du débat à travers l'expression de «ville garantie ». Sous la pression d'un libéralisme normalisateur, la gouvernance urbaine s'appuierait sur un réseau d'experts techniques (architectes, aménageurs, agences de certification) pour concevoir un design urbain conforme aux standards internationaux et aux exigences actuelles de qualités (esthétique, sécuritaire, écologique) des aménagements. Dans ce mouvement mondial de certification de la qualité des villes, la création d'ambiances urbaines consensuelles, déployant les registres de l'enchantement, de l'apaisement assure le pouvoir d'attractivité des villes en même temps qu'une illusion de convivialité, d'ouverture à la différence et d'adhésion aux valeurs déployées par la gouvernance libérale. À travers l'exemple des villes méditerranéennes (2018) et du cas de Lisbonne en particulier (2019), Breviglieri montre comment la scénarisation des espaces urbains - outre qu'elle appauvrit les ambiances en neutralisant les situations de trouble - rend problématique l'accueil de l'étrangeté et de la discordance. À travers l'application d'un " vernis cosmopolite ", c'est en effet «le seuil de tolérance à la différence gênante qui est particulièrement abaissé " (Breviglieri, 2018, p. 7). La partition des espaces en fonction des types d'usages attendus est aussi accrue. 
15 La critique est acerbe : elle porte sur le pouvoir normalisateur des ambiances urbaines et sur sa capacité à se jouer d'une réalité préexistante, diversifiée, inscrite dans l'histoire.

16 S'il est moins frontalement question d'ambiances urbaines dans les travaux de Luca Pattaroni, l'articulation de cette question à celle de la composition architecturale d'un ordre urbain affleure en particulier dans son enquête sur le quartier des Grottes à Genève (2013).

17 Les Grottes ont constitué, dès le début des années 1970, un laboratoire de la culture alternative genevoise. De nouvelles formes du vivre-ensemble y ont été expérimentées d'abord en réaction contre des politiques de rénovation urbaine accusées de reproduire un ordonnancement des territoires aveugle à la différence, puis au nom de revendications en faveur de la création de lieux culturels autonomes. Pattaroni décrit ainsi en quoi l'adoption de formes de vie collectives et autogérées, attentives à une qualité de vie devenue cause commune, a soutenu en pratique des valeurs morales (de solidarité, d'accueil, de partage, de créativité...) édifiées en réaction à l'ordre établi. La critique habitante est ici discursive autant qu'expérientielle. Elle prend forme au travers de pétitions, d'occupations illégales, de formes d'expressions politicoartistiques et d'un soin quotidien apporté au cadre de vie. Cette critique s'ancre aussi dans un fort sentiment d'appartenance au quartier, revendiqué et largement fécondé par la participation à la création d'une ambiance conviviale. Pattaroni $(2013,2021)$ ne l'exprime pas ainsi, mais cet attachement à la convivialité des ambiances constitue un levier de mobilisation fort pour les luttes urbaines autant qu'un élément central de la cohésion et de l'émancipation du groupe.

18 La négation de la "valeur d'usage " des espaces par les politiques urbaines, ou sa revendication par les habitants, traverse en arrière-plan ces travaux.

Dans son analyse du "capitalisme esthétique», Gernot Böhme (2017b) déplace le problème en même temps qu'il l'ancre plus fortement dans le champ des atmosphères. S'il partage ce constat d'une montée en puissance d'une " économie esthétique » fondée sur la production d'atmosphères marchandes, il propose d'en interroger davantage « la valeur de mise en scène ». Selon lui, les atmosphères sont susceptibles d'orienter les dispositions affectives des individus et de modifier en profondeur leur vécu. Il s'agit alors non plus de dévoiler et de juger par la négative cette esthétisation généralisée du quotidien - que Böhme juge par ailleurs ancienne et légitime - ni même de se contenter de la décrire, mais plutôt de rendre compte de l'emprise des atmosphères sur nos manières de sentir et de se sentir: "[ce pouvoir des atmosphères] influe sur les dispositions des êtres humains, il se saisit de leurs humeurs. [...] Il est toutefois invisible et plus difficile à appréhender que d'autres formes de violence » (Böhme, 2018, p. 40). Critiquer reviendrait à reconnaitre la nécessité qu'ont les êtres humains de fabriquer des environnements dans lesquels ils se sentent bien et peuvent être mis en scène tout en questionnant la capacité (d'aliénation, d'aveuglement, de manipulation) des atmosphères ${ }^{3}$. Cette tâche constituerait le devoir d'une esthétique des atmosphères, dans le sens où elle aurait à porter attention à la manière dont la vie ordinaire se déroule et est vécue, permet à chacun de prendre une place, de donner sens à ses actions, d'entrer en négociation avec autrui.

Céline Bonicco-Donato (2016) nous invite également à une réflexion philosophique sur les rapports entre pouvoir et ambiance. Mais elle se positionne à l'interface des 
propositions de Gernot Böhme et d'une sociologie urbaine critique d'inspiration marxiste.

21 La relecture des travaux de Michel Foucault (2004) sur l'évolution des «technologies gouvernementales du pouvoir» enrichit chez elle une pensée de l'urbanité qui n'oppose pas vision disciplinaire et vision émancipatrice de la société, mais considère le potentiel d'hospitalité des ambiances à l'heure du néolibéralisme. Proposition est alors faite de considérer le design ambiantal comme un outil insidieux de régulation et de contrôle des stimuli sensible des individus autant que comme un support possible pour des pratiques de détournement et de résistance. Cette proposition pour « une philosophie politique des ambiances » se construit dans un souci d'apport au champ de la conception et de l'aménagement urbain. Elle se différencie en cela des thèses précédemment évoquées. L'auteure prolonge les réflexions sur le lissage des ambiances urbaines et propose d'en examiner les effets sur le gouvernement des conduites sociales. Son objectif est de comprendre comment des qualités d'ambiances prédonnées participent d'une plus ou moins grande félicité de la vie urbaine ordinaire.

\section{Renouveler les postures épistémologiques dans le champ des ambiances}

Les transformations programmées des ambiances urbaines sont donc appréhendées et dénoncées ici comme des effets diffus des politiques néolibérales, comme des projections insidieuses de valeurs nouvelles et de relations de pouvoir instituant un nouveau " partage du sensible " (Rancière, 2000) et éprouvant l'hospitalité minimale de la ville (Joseph, 1998). A contrario de la critique implicite qui autrefois émaillait les travaux dans le champ des ambiances, c'est aujourd'hui le surinvestissement des sens plutôt que leur négligence - et leur articulation à des desseins esthétiques, marchands, de manipulation affective qui font l'objet des reproches explicites adressés au champ de la conception et de l'aménagement urbain. Pour exemple, les ouvrages de Christian Borch (2014) sur le marketing sensoriel ou de Gilles Lipovetsky et Jean Serroy (2013) sur l'esthétisation du monde décrivent le poids grandissant de cette "atmosphérisation » de l'architecture et des villes sur la vie quotidienne, en insistant sur l'hyper-hédonisme des existences et la quasi sacralisation du jeu des apparences qu'elle engendre. C'est aussi une inquiétude quant à la normalisation des ambiances urbaines et à l'avènement de formes nouvelles d'emprise qui conduit les analyses.

Féconds, ces travaux peinent toutefois à articuler, dans un même programme de travail, une critique sociale et politique à l'œuvre dans le champ de la sociologie urbaine critique et des critical urban studies et une critique ancrée dans le champ des ambiances urbaines, attentive aux modalités concrètes et affectives de l'expérience urbaine ordinaire. Lorsque c'est le cas - chez Gernot Böhme et Céline Bonicco-Donato particulièrement - les efforts conceptuels restent à ancrer dans des situations d'enquête qui en nuanceraient les hypothèses.

La proposition faite ici consiste à construire cette critique sociale depuis le champ des ambiances en clarifiant et en débattant - à partir d'enquêtes situées - des types de modes d'existence que permettent ou entravent ces transformations programmées des ambiances urbaines, qu'elles relèvent de l'avènement du néolibéralisme ou de toute autre stratégie de configuration de nos cadres de vie. Comme l'ont montré Erving 
Goffman (2013 [1963]), et à sa suite Isaac Joseph $(1995,1998)$, ces cadres matériels et moraux organisent les manières dont nous nous engageons dans les situations urbaines et le cours ordinaire des interactions en public. Mais ils sont aussi "ambiantaux", s'incarnent dans nos sensibilités, nos manières d'être et de juger. Rendre compte de cet enchevêtrement sur un mode critique requiert alors de ne pas seulement travailler sur les effets des politiques d'ambiance sur les conduites sociales, mais de mener une enquête ethnographique sur la façon dont les citadins en font l'expérience, sont plus ou moins éprouvés, les endossent ou pas. Comblant un manque dans les travaux à partir des ambiances, une telle critique questionne également leur part dans la création de formes de stigmatisation ou de vulnérabilité en ville.

Ce programme de travail partage donc bien la perspective écologique au fondement de la problématique des ambiances (Amphoux, 1998 et al. ; Augoyard, 1995; Thibaud, 2013). Mais il l'élargit en abordant de front et sans préjuger de leur négativité la valeur de ces ambiances programmées. Trois perspectives de travail sont proposées :

\section{Saisir du politique dans les ambiances}

26 La première consiste à politiser les ambiances en considérant que si elles relèvent bien d'une imbrication entre l'individuel (voire l'intime) et le collectif, elles sont aussi porteuses d'enjeux publics :

Le politique [...] peut se nicher dans les conditions de possibilité de l'expérience d'un vivre ensemble - cette constitution d'usages, de croyances et de mœurs, de manières de voir, de dire et de faire, qui fait lien dans la vie commune [...]. (Cefaï, 2011, p. 545)

Ancré dans une écologie matérielle et sociale, le politique surgit aussi dans une gradualité de tonalités affectives qui infléchit l'expérience urbaine ordinaire et l'expressivité des situations. Éric Chauvier (2018) fait valoir cet horizon politique des ambiances en pointant du doigt l'injonction à la positivité qu'elles contiendraient implicitement : les jeux d'ambiance actuels sont des «mises en scène ponctuelles de la vie sociale " (p. 101) qui voilent l'indigence et rendent imperceptible toute situation dissonante. Reprenons son exemple: la présence d'une famille de réfugiés assise à même le bitume, dans une rue marchande où l'aura des ambiances est prégnante, entache leur positivité, mais ni ne « casse l'ambiance » ni ne nous empêche de « passer notre chemin » avec indifférence. Cette partition des ambiances - Chauvier remet en cause l'idée d'un continuum déjà là - dessinerait des situations plaisantes et d'autres « dominées par l'inexpressivité et où la possibilité d'une condition ambiantale de félicité n'est pas ratifiée » (p. 105). Si la thèse peut paraitre duale - l'ambiance ne serait que du côté du ravissement et de l'expressivité des situations - elle ouvre une perspective de travail dont une critique de l'urbain depuis le champ des ambiances pourrait se saisir : celle d'un questionnement sur la disparition potentielle de styles d'ambiance et, avec elle, de style d'existence. Que fabrique-t-on aujourd'hui comme conditions de possibilité ambiantale de l'expérience urbaine ordinaire? Quelles sont les ambiances qui s'éteignent ou risquent de s'éteindre et celles qui, au contraire, apparaissent ? En quoi et comment les processus de normalisation, de contrôle et de mise en scène des ambiances modifient-ils l'intensité des ambiances et, avec elle, celle de la vie sociale ordinaire? le politique dans nos manières d'agir, mais aussi "d'être affecté ». Ces manières 
relèvent autant de l'intime que du partagé et, là encore, les tonalités d'ambiance ne sont pas neutres.

Ce qui est éprouvé dans le cours même de l'expérience - l'embarras, la gêne, l'aisance, le contentement...- transparaît à même les corps, dans des rythmes et des allures, dans des regards et des gestes... Or cette ambiance, incarnée dans la situation, limite ou active, selon des degrés et des temporalités divers, la capacité d'agir et de réagir des individus en présence. Elle invite à se conformer aux attentes et à se soucier ou pas d'autrui. Elle rend certaines causes plus ou moins dignes d'un intérêt commun et d'un engagement, solidifie ou fragilise un groupe. À une échelle préréflexive, elle participe d'une inflexion dans le temps des "modalités mêmes de l'acte d'exister " au-delà du travail de figuration de chacun (Boukir, 2018).

Il y a, autrement dit, à mettre en exergue l'importance d'une écologie affective dans l'évolution conjointe des façons de ressentir, d'exister et d'être-ensemble. Suivre cette piste amène à considérer le politique moins comme le gouvernement des hommes - et un gouvernement qui engendrerait des relations de domination entre les plus forts et les plus faibles - que comme l'espacement par où se compose, depuis les places et les sensibilités de chacun, des manières d'être et d'être affecté partagées. Saisir du politique dans les ambiances conduit alors à observer et à décrire finement la manière dont des styles d'existence sont affectés par des changements d'ambiance. Cette description est critique non pas parce qu'elle dévoile et condamne des régimes de contrôle ou de hiérarchie, mais parce qu'en décrivant des sensations éprouvées dans le cours même des situations, en racontant des impressions vécues, elle dit ce qui entrave ou favorise la possibilité de prendre part à une expérience partagée. Elle est critique parce qu'elle donne à saisir l'épaisseur sensible de l'expérience et que c'est depuis cette épaisseur qu'elle interroge toutes les épreuves qui la traversent.

\section{Historiciser la chronicité des mutations d'ambiance}

31 Une telle critique ne peut toutefois pas s'abstraire des arrière-plans historiques, sociaux et culturels - voire des chocs d'ambiance (Fuzessery \& Simay, 2008) - dans lesquels les expériences urbaines ont lieu et évoluent. L'histoire des sensibilités le montre (Elias, 1939 ; Corbin, Courtine \& Vigarello, 2016, 2017), les mutations de l'urbain se déploient dans des histoires qui pèsent sur l'ambiance, sur les sensibilités humaines, sur l'évolution des appareils perceptifs et qui obligent le citadin, à chaque époque, à s'adapter à de nouveaux cadres de vie.

La problématique des ambiances architecturales et urbaines a longtemps ignoré cette chronicité des mutations, trop attachée à la saisie hic et nunc de la phénoménalité de l'espace construit. La plupart des enquêtes menées dans ce champ donnent lieu à des monographies d'espaces qui, lorsqu'elles restituent leurs variations d'ambiance, font peu de cas de leur épaisseur historique ${ }^{4}$. Les ambiances décrites sont celles d'espaces publics ou de quartiers saisies à certaines heures du jour ou de la nuit, à certaines saisons ou lors d'évènements marquants de la vie urbaine. Rares sont celles qui s'appuient sur une saisie au long cours des modulations d'ambiances et de leurs effets sur l'expérience et les sensibilités.

Une telle perspective de travail - que nous pourrions qualifier de « anhistorique » - ne pose aucun problème si l'on considère que les terrains étudiés ne sont pas agités par des basculements de modèles de société ou par des évolutions plus ou moins 
perceptibles qui s'enracinent dans l'Histoire (ou dans des histoires) et qui bouleversent les cours d'expérience (Masson \& Thomas, 2013). Mais à partir du moment où les terrains d'enquête confrontent directement le chercheur à des crises - ou bien lorsque, avec d'autres, il partage la sensation que « quelque chose se passe »-comment faire?

Pour répondre à cette interrogation, nous émettons l'hypothèse de la portée critique d'une approche historicisée des évolutions des ambiances urbaines. Saisir l'épaisseur historique des terrains - en faisant la biographie sociale et culturelle de leurs ambiances (Pecqueux, 2012) - peut être une manière de mettre en perspective les mutations profondes des cadres de l'expérience urbaine et la façon dont elles modifient les façons de sentir et d'être des citadins ${ }^{5}$. Retraçant les itinéraires intellectuels de Simmel, Kracauer et Benjamin, Stéphane Fuzessery et Philippe Simay (2008) reviennent précisément sur la dimension historique et incarnée des "chocs " d'ambiance: les mutations physiques, aménagées et esthétiques des grandes métropoles urbaines transforment sur le long terme les styles de vie urbains et, avec eux, le caractère des citadins et les formes visuelles de gestion des coprésences en public. Reprendre cette hypothèse ne conduit pas à renoncer au positionnement naturaliste au fondement de la problématique des ambiances, en prônant l'importance de la grande histoire sur la petite et en renonçant à l'étude de l'ordinaire des situations publiques: observer et décrire le monde urbain tel qu'il est en train de se faire et de se vivre constitue bien une voie féconde pour dépasser des conceptions figées.

Cette hypothèse de travail permettrait aussi de s'affranchir des prises de position dualistes, prononcées à chaque époque, à l'égard des mutations des sociétés urbaines.

Un bref détour par la littérature dans le domaine montre en effet un clivage entre vision pessimiste et vision optimiste de ces transformations. La première dénonce les pathologies de l'architecture et des ambiances, leur rôle dans l'appauvrissement des expériences et le désenchantement du citadin (Mumford, 2001; Sennett, 2002; Pallasmaa, 2010 ; Koolhaas, 2011). La seconde loue la façon dont elles ré-enchantent la vie urbaine (Roux, 2002; Garnier, 2008; Winkin \& Lavadinho, 2008). Construire l'histoire sensible des rapports entre les sociétés humaines et les ambiances permettrait de tempérer une critique souvent nostalgique, parce que guidée par la disparition du familier et par une vision idéalisée du passé. Car l'envie de faire revivre ce passé conduit bien souvent à nier la possibilité même que de nouvelles sensibilités et formes d'urbanité puissent émerger. Pourtant, si l'on en croit Simmel et Benjamin :

[...] l'hyperstimulation sensorielle et l'état de choc contiennent un potentiel libérateur pour le citadin. Pour Simmel, le nouveau mode de vie citadin peut être lu comme une parade - à bien des égards émancipateur - opposée par l'habitant des grandes villes aux nouvelles conditions de perceptions générées par la métropolisation [...]. Ils contribuent même, selon Benjamin, à accroître les facultés perceptives de l'homme moderne (Füzessery \& Simay, 2008, p. 16).

Historiciser la chronicité des mutations d'ambiance permettrait finalement de comprendre comment des régimes d'affects se sédimentent au long cours, voire s'institutionnalisent, au travers d'un travail politique d'appréciation collective, luimême force d'action (Bantigny, Cohen \& Gobille, 2020). Doté d'une puissance documentaire, un tel geste se doublerait d'une puissance d'interpellation en réhabilitant les modes d'existence des ambiances et leur opérationnalité sur les formes sensibles de la vie urbaine. 


\section{S'engager en chair et au long cours dans l'enquête} reposé sur des dispositifs de mise en situation de collectifs de chercheurs qui n'ont laissé que peu de place à l'observation située des formes de vie sensibles des usagers. Ce fut le cas des travaux précédemment cités interrogeant le poids de certaines politiques de réaménagement des mobilités urbaines sur les manières de se mouvoir et d'êtreensemble en public : sur la Redoma de Petare à Caracas (Venezuela) comme à Porto do Barra à Salvador da Bahia (Brésil), diverses marches urbaines ont engagé des collectifs de chercheurs, de nationalités différentes, pour mieux comprendre comment les idéologies de la fluidité ou de la pacification se concrétisaient in situ et s'incarnaient (Fiori \& Thomas, 2016). Dans ce cadre de travail, "l'immersion par corps des chercheurs » (ibid.), en les dé-familiarisant et en mobilisant leur sens, renouvelle leurs modes d'appréhension et d'analyse des terrains : les corps, tels qu'en prise et affectés par les ambiances, sont des instruments d'intelligibilité de leur efficace. Ils donnent accès à des registres de l'expérience urbaine qui, s'ils n'avaient pas été mobilisés, seraient passés inaperçus ${ }^{6}$.

41 S'engager en chair est différent. La chair ne renvoie pas au corps physique mais à l'expérience préréflexive que nous avons de notre présence au monde (Merleau-Ponty, 1964) et aux ambiances. S'engager en chair suppose un mode d'être au terrain singulier: demeurer en rapport constant avec autrui et ce que nous ressentons en commun, au point d'être traversés sensuellement par des épreuves physiques et affectives. Ces épreuves, elles ne sont plus seulement un corpus de l'enquête. Elles sont un moteur en même temps qu'un instrument de " ce qui se joue » dans l'expérience en cours. "Mieux saisir cette expérience sensible du politique, au plus près des mots, des corps et des gestes" apparait alors fondamental (Bantigny, Cohen \& Gobille, 2020, p. 20). 

dans la quotidienneté des usagers et les nuances de tonalités qui la fabriquent. Cet engagement conduit souvent à des expériences troublantes. Il fragilise parce qu'il remet en cause ce qui est acquis, oblige à des désapprentissages et à des repositionnements continuels. Et c'est en cela qu'il est critique. En renforçant la présence aux ambiances et à leurs inflexions discrètes, il étaye une intelligence charnelle à cette toile de fond de nos expériences. Il modifie les impressions et, avec elles, les manières de voir, d'entendre. Il permet, dans la durée, de saisir des variations parfois diffuses et difficilement verbalisées, d'accorder une importance égale aux êtres et aux choses, aux phénomènes sensibles comme aux interactions, au matériel comme aux intensités. Dans l'exemple cité précédemment, la répétition des marches collectives sur un temps relativement long ( 3 à 4 ans) a permis de saisir et de rendre intelligibles les épreuves (pratiques, perceptives et affectives) d'accommodement des chercheurs aux diverses circonstances auxquelles leurs parcours quotidiens les exposaient au sein de la Redoma de Petare ou de Porto do Barra (Fiori \& Thomas, 2013). Dans le même temps, le partage de ces expériences a conduit les participants - chercheurs, mais aussi professionnels de l'aménagement urbain et politiques à Caracas - à renouveler leurs représentations des terrains - jusqu'ici limitée à la figure du chaos - et donc à poser les bases d'un débat sur les enjeux de leurs transformations. Dans ce cas, comme ailleurs, le long cours transforme les vécus de chacun, obligeant le chercheur à toujours se déplacer vers le point de vue ou le point d'écoute de l'Autre. Et c'est précisément en cela qu'il constitue une ressource pour l'enquête critique.

\section{Critiquer : documenter (aussi) l'imperceptible, apprécier affectivement, interpeller ${ }^{7}$}

Ce projet de fonder une critique de l'urbain depuis les ambiances ouvre donc la voie à une réorientation des problématiques de ce champ vers un questionnement sur les enjeux (sociaux, culturels, éthiques, moraux...) liés à leur transformation programmée. La proposition de saisir du politique dans les ambiances en constitue l'argument central.

L'enjeu d'une telle critique ne consiste ni à déplorer l'existence d'un marketing des ambiances plus propice à valoriser l'image d'une ville qu'à en bonifier les conditions d'expérience ni à supposer un contrôle des corps et des conduites urbaines depuis une instrumentalisation du sensible. À la différence des approches critiques en sciences sociales, il ne s'agit pas non plus de lever le voile sur les mécanismes cachés de la domination sociale et spatiale (Fossier \& Manicki, 2007). Une critique de l'urbain depuis le champ des ambiances cherche davantage à comprendre les articulations réciproques entre l'ambiance et le politique. Elle a pour enjeu principal de comprendre et de décrire - au plus près des situations concrètes - la manière dont, dans certaines configurations, les citadins développent leurs capacités d'action, composent avec les éléments de leur environnement, ont prise sur leur milieu de vie, le façonne en retour.

Mais critiquer consiste également à qualifier les manières dont nous pâtissons ensemble des conditions d'ambiance dans lesquelles nous nous mouvons. Certaines situations sont plus problématiques que d'autres et font parfois s'effondrer notre sentiment d'appartenance à un groupe ou notre capacité à accueillir l'Autre. D'autres situations nous fragilisent et mettent en défaut nos capacités à prendre part à « ce qui se passe ». L'expérience récente du confinement et les incertitudes liées à la crise 
sanitaire bouleversent notre quotidien, modifient nos manières de vivre et de nous comporter en public, participent de nouvelles formes de vulnérabilité renouvelées. La critique depuis le champ des ambiances se préoccupe de saisir ces variations de tonalités affectives qui se manifestent bien souvent en deçà d'une visibilité et/ou d'une énonciation claire, dans des gestes, des états de corps, des styles d'existence particuliers. Elle prend la mesure de ces difficultés, sans toutefois adopter la posture dénonciatrice souvent mobilisée par les approches critiques. Elle évalue davantage - au sens d'apprécier affectivement - "ce qui pose problème" dans les situations et l'expérience urbaine.

De ce point de vue, une critique de l'urbain depuis le champ des ambiances participe de l'enquête. Mais elle le fait à partir de deux postures particulières: historiciser la chronicité des mutations d'ambiance; s'engager en chair et au long cours dans l'enquête. Elle ne renoue donc pas avec des formes de pensées nostalgiques qui idéaliseraient le passé, mais produit de nouveaux contextes d'intelligibilité des transformations des ambiances à partir de leur généalogie. En complément de cette historicisation, elle déploie des protocoles d'enquête qui permettent une saisie en situation de l'épaisseur et de la complexité des expériences sensibles ordinaires. Ces protocoles engagent le chercheur dans une présence renouvelée sur le terrain, indispensable à la re-contextualisation des observations et des histoires. Mais ils l'engagent en chair, en tant qu'il est un individu doté de sens et de valeurs, susceptible d'être concerné et éprouvé par les troubles d'autrui, capable de les documenter et de les décrire finement afin de faire émerger des interrogations à propos de "ce qui se passe ", à même des situations concrètes et ordinaires d'existence.

La critique depuis le champ des ambiances opère enfin en tant que puissance d'interpellation. L'appréciation émise sur les situations révèle notre attachement à certaines formes de vie et notre inquiétude lorsqu'elles sont bousculées, ce "notre " articulant autant le registre habitant que celui du chercheur. Elle n'est en rien l'expression d'un avis final et définitif, formulé par une autorité reconnue (l'expert, le scientifique), qui viendrait approuver ou à l'inverse condamner une démarche, une pratique et le groupe qui les porte. La critique invite à exprimer des désirs communs quant au devenir sensible de l'urbain, plutôt qu'à développer des propositions prescriptives. Elle incite à se soucier de l'avenir en faisant valoir « ce qui nous touche " (Hache, 2011).

\section{BIBLIOGRAPHIE}

Adey, Peter et al.. 2013. Pour votre tranquillité : Ambiance, atmosphere, and surveillance. Geoforum. n 49, p. 299-309. [en ligne] Disponible sur : https://doi.org/10.1016/j.geoforum. 2013.04.028 (consulté en mars 2014)

Anderson, Ben. 2009. Affective Atmospheres. Emotion, Space and Society. 2 (2), p. 77-81. [en ligne] Disponible sur : https://doi.org/10.1016/j.emospa.2009.08.005 (consulté en février 2010) 
Amphoux, Pascal et al.. 1998. La notion d'ambiance. Une mutation de la pensée urbaine et de la pratique architecturale. Paris : PUCA.

Amphoux Pascal, Chelkoff Grégoire, Thibaud Jean-Paul. 2004. Ambiances en débat. Bernin : À la Croisée.

Atienza, Ricardo et al.. 2007. Les dimensions émergentes de l'intimité en dehors du chez-soi dans les zones d'habitat individuel dense. Paris : PUCA. Grenoble : CRESSON.

Augoyard, Jean-François. 2011. Faire une ambiance. Introduction. In : Augoyard, Jean-François (ed.). 2011. Faire une ambiance: Actes du $1^{\text {er }}$ congrès international sur les ambiances. Grenoble : RIA, ENSAG. Bernin : À la Croisée, p. 17-35.

Augoyard, Jean-François. 2000. Ambiances et nuisances : les ambiances urbaines entre technique et esthétique. In : Peyretti Guy \& Prost Thierry (eds). Une décennie de génie urbain : connaissances nouvelles, acteurs de la décision, communauté scientifique du génie urbain. Certu, Ministère de l'Équipement, des Transports et du Logement, pp. 69-77.

Augoyard, Jean-François. 1998. Éléments pour une théorie des ambiances architecturales et urbaines. Les Cahiers de la Recherche Architecturale et Urbaine. $\mathrm{n}^{\circ}$ 42-43. p. 7-23.

Augoyard, Jean-François. 1995. L'environnement sensible et les ambiances architecturales. L'Espace Géographique. $\mathrm{n}^{\circ}$ 4. p. 302-318.

Augoyard, Jean-François. 1991. La vue est-elle souveraine dans l'esthétique paysagère ? Le Débat. $n^{\circ}$ 65. p. 51-58.

Balaÿ, Olivier. 2013. L'ambiance comme objet historique. In : Beck Robert, Krampl Ulrike \& Retaillaud-Bajac, Emmanuelle (eds.). Les cinq sens de la ville du Moyen âge à nos jours. Tours, Presses Universitaires François Rabelais, pp. 29-51

Bantigny Ludivine, Cohen Déborah, Gobille Boris. 2020. La chair du politique. Sensibilités. Histoire, critique \& sciences sociales. $\mathrm{n}^{\circ}$ 7. Paris : $\mathrm{CNL}$, Anamosa.

Benjamin, Walter. 2000 [1939]. L'œuvre d'art à l'époque de sa reproductibilité technique in Euvres III. Paris : Gallimard.

Bissel, David. 2010. Passenger Mobilities: Affective Atmospheres and the Sociality of Public Transport. Environment and Planning D: Society and Space. 28 (2), p. 270-289. [en ligne] Disponible sur : https://doi.org/10.1068/d3909 (consulté en août 2010)

Böhme, Gernot. 2020. Aisthetique. Pour une esthétique de l'expérience sensible. Dijon : Presses du réel. Böhme, Gernot. 2017a. The aesthetic of atmospheres. Edited by Jean-Paul Thibaud. London : Routledge.

Böhme, Gernot. 2017b. Critique of aesthetic capitalism. S.I : Mimesis International.

Böhme, Gernot. 2018. L'atmosphère : fondement d'une nouvelle esthétique. trad. Maxime Le Calvé. Communications : Exercices d'ambiance. 102, p. 25-49. [en ligne] Disponible sur : https:// www.cairn.info/revue-communications-2018-1-page-25.htm (consulté en janvier 2021)

Bonicco-Donato, Céline. 2016. De l'aménagement des milieux à la technologie ambiantale du pouvoir. Éléments pour une philosophie politique des ambiances. In : Rémy, Nicolas \& Tixier, Nicolas (eds). 2016. Ambiances, demain : actes du $3^{e}$ congrès international sur les ambiances. Grenoble : RIA, ENSAG. p. 593-598.

Borch, Christian. 2014. Architectural Atmospheres. On the experience and politics of architecture. Bâle : Birkhäuser Verlag GmbH. 
Boukir, Kamel. 2018. Les ambiances de la mort. In : Le Calvé, Maxime \& Gaudin, Olivier (eds). Exercices d'ambiances. Communications. $n^{\circ} 102$. p. 169-184. [en ligne] Disponible sur : https:// www.cairn.info/revue-communications-2018-1-page-169.htm (consulté en février 2021)

Brenner Neil, Marcuse Peter \& Mayer Margit. 2009. Cities for people, not for profit. City. $n^{\circ} 13-2$, p. $176-184$

Breviglieri, Marc. 2019. Lisbonne, $21^{\mathrm{e}}$ siècle. EspaceTemps.net. [en ligne] Disponible sur : https:// www.espacestemps.net/articles/lisbonne-21e-siecle/ (consulté en janvier 2020)

Breviglieri, Marc. 2018. L'affadissement des villes méditerranéennes et la désacralisation de la figure de l'hôte. Sociologies : Hospitalités. L'urgence politique et l'appauvrissement des concepts. [en ligne] Disponible sur : http://journals.openedition.org/sociologies/6821 (consulté en janvier 2020)

Breviglieri, Marc. 2013. Une brèche critique dans la ville garantie. Espaces intercalaires et architectures d'usage. In : Cogato-Lanza, Elena et al. (eds). De la différence urbaine : le quartier des Grottes / Genève. Genève : MetisPresses. p. 213-236.

Cefaï, Daniel. 2011. Vers une ethnographie (du) politique : décrire des ordres d'interaction, analyser des situations sociales. In : Berger, Matthieu et al (eds). Du civil au politique. Ethnographies du vivre-ensemble. Bruxelles : Peter Lang. p. 545-598.

Chauvier, Éric. 2018. Le concept d'« ambiance » à l'épreuve de la vie ordinaire. In : Le Calvé, Maxime \& Gaudin, Olivier (eds). Exercices d'ambiances. Communications. $n^{\circ} 102$. p. 99-109. [en ligne] Disponible sur : https://www.cairn.info/revue-communications-2018-1-page-99.htm (consulté en février 2021).

Chelkoff, Grégoire. 2005. De l'espace à l'ambiance : formes sensibles de l'architecture et transformations de l'environnement urbain. HDR en urbanisme. IUG, CRESSON.

Chelkoff, Grégoire \& Thibaud, Jean-Paul. 1992. L'espace public, modes sensibles : le regard sur la ville. Les Annales de la Recherche Urbaine. ${ }^{\circ}$ 57-58. p. 7-16.

Corbin Alain, Courtine Jean-Jacques \& Vigarello Georges (dirs). 2016. 2017. Histoire des émotions. 3 tomes. Paris, Seuil.

Edensor, Tim. 2015. Producing atmospheres at the match: Fan cultures, commercialization and mood management in English football. Emotion, Space and Society. 15, p. 82-89. [en ligne] Disponible sur : https://doi.org/10.1016/j.emospa.2013.12.010 (consulté en mai 2016)

Edensor, Tim \& Sumartojo, Shanti. 2015. Designing Atmospheres: introduction to Special Issue. Visual Communication. 14 (3), p. 251-265. [en ligne] Disponible sur : https://doi.org/ 10.1177/1470357215582305 (consulté en décembre 2015)

Elias, Norbert. 1994 [1939]. La civilisation des mœurs. Paris : Calmann-Lévy.

Fiori, Sandra \& Garcia Sanchez, Pedro. 2014. Les espace-temps de la mobilité labile. In : Thomas, Rachel (dir.) MUSE : les énigmes sensibles des mobilités urbaines contemporaines. Grenoble : CRESSON. ANR 10-ESVS-0013. p. 91-161.

Fiori, Sandra \& Thomas, Rachel. 2016. L'immersion par corps des chercheurs. Expérimentations méthodologiques à Salvador da Bahia et Caracas. Corps. Revue interdisciplinaire. $\mathrm{n}^{\circ} 14$, p. 229-237. [en ligne] Disponible sur : https://www.cairn.info/revue-corps-2016-1-page-229.htm (consulté en janvier 2017)

Fiori, Sandra \& Thomas, Rachel. 2013. Être piéton à Salvador de Bahia et Caracas : une proximité fragilisée ? Géoregards. n 6, p. 83-96. 
Fossier, Arnaud \& Manicki, Anthony. 2007. Où en est la critique ? Tracés. Revue de sciences humaines. $n^{\circ} 13$, p. 5-22. [en ligne] Disponible sur : https://journals.openedition.org/traces/306 (consulté en janvier 2008)

Foucault, Michel. 2004. Naissance de la biopolitique. Paris : EHESS/Seuil/Gallimard.

Fuzessery, Stéphane \& Simay, Philippe (eds). 2008. Le choc des métropoles. Simmel, Kracauer, Benjamin et la GroBstadt. Paris : Ed. de l'Éclat.

Garnier, Jean-Pierre. 2008. Scénographies pour un simulacre : l'espace public réenchanté. Espaces et Sociétés. $n^{\circ} 134$, p. 67-81. [en ligne] Disponible sur : https://doi.org/10.3917/esp.134.0067 (consulté en mars 2009)

Goffman, Erving. 2013 [1963]. Comment se conduire dans les lieux publics. Notes sur l'organisation sociale des rassemblements. Trad. Cefaï, Daniel. Paris : Économica.

Guha, Ranajit \& Gayatri Spivak (eds). 1988. Selected Subaltern Studies. New York : Oxford University Press.

Hache, Émilie. 2011. Ce à quoi nous tenons. Paris : La Découverte

Hadbi, Ryma. 2020. Révéler ce qui fait patrimoine pour penser le renouvellement urbain des cités d'habitations à bon marché de Grenoble. Les Cahiers de la recherche architecturale, urbaine et paysagère, Ministère de la Culture. [en ligne] Disponible sur : https://doi.org/10.4000/craup.4606 (consulté en octobre 2021)

Harvey, David. 2011. Le capitalisme contre le droit à la ville : néolibéralisme, urbanisation, résistances. Trad. Cyril Le Roy, Nicolas Vieillescazes, Clémence Garrot \& Joséphine Gross. Paris : Éditions Amsterdam.

Harvey, David. 1973. Social Justice and the City. Londres, Arnold.

Imbroscio, David \& Davies, Jonathan S. 2010. Critical urban studies. New directions. New York : State University of New York Press.

Joseph, Isaac. 1998. La ville sans qualités. Paris : l'Aube.

Joseph, Isaac. 1995. Prendre place. Espace public et culture dramatique. Paris : Ed. Recherches.

Juan Cantavella, Anna. 2009. Espace urbain, art et utopie : une approche critique de la dimension utopique dans l'artiation des espaces urbains de la ville. Thèse de doctorat en urbanisme. Grenoble : Université de Grenoble ; Barcelone : Université de Barcelone.

Koolhaas, Rem. 2011. Junkspace. Repenser radicalement l'espace urbain. Paris : Payot.

Lefebvre, Henri. 1947. Critique de la vie quotidienne. Tome I: Introduction. Paris : l'Arche.

Leroux, Martine \& Bardyn, Jean-Luc. 2003. A l'écoute de l'hôpital. Grenoble : CRESSON. Paris : APHP. Lipovetsky, Gilles \& Serroy, Jean. 2013. L'esthétisation du monde. Vivre à l'âge du capitalisme artiste. Paris : Gallimard.

Margier, Antonin. 2017. Cohabiter l'espace public. Conflits d'appropriation et rapports de pouvoir à Montréal et Paris. Rennes : PUR.

Masson, Damien et al.. 2014. Surveillance(s) en public. MUSE : les énigmes sensibles des mobilités urbaines contemporaines. In : Thomas, Rachel (dir.) MUSE : les énigmes sensibles des mobilités urbaines contemporaines. Grenoble : CRESSON. ANR 10-ESVS-0013. p. 165-245.

Masson, Damien \& Thomas, Rachel. 2013. Towards an 'ambiance-grounded' critique? RGS-IBG Annual International Conference. Londres, 29 août. 
Massumi, Brian. 2015. Politics of affects. Cambridge : Polity Press.

McCormack, Dereck. 2008. Geographies for Moving Bodies : Thinking, Dancing, Spaces. Geography Compass. 2 (6), p. 1822-1836. [en ligne] Disponible sur : https://doi.org/10.1111/j.

1749-8198.2008.00159.x (consulté en juin 2011)

Merle, Isabelle. 2004. Les Subaltern Studies. Retour sur les principes fondateurs d'un projet historiographique de l'Inde coloniale ». Genèses. n 56, p. 131-147. http://www.cairn.info/revuegeneses-2004-3-page-131.htm (consulté en avril 2016)

Merleau-Ponty, Maurice. 1964. Le visible et l'invisible. Textes réunis par Claude Lefort. Paris : Gallimard.

Mumford, Lewis. 2001 [1961]. Le piéton de New York. Paris : Éd. du Linteau.

Pallasmaa, Juhani. 2010. Le regard des sens. Paris : Éd. du Linteau.

Pattaroni, Luca (dir). 2021. La contre-culture domestiquée : art, espace et politique dans la ville gentrifiée. Genève : MétisPresses.

Pattaroni, Luca. 2013. Politiques de la différence. Critique et ouverture des possibles dans l'ordre de la ville. In : Cogato Lanza Elena, et al. (eds). De la différence urbaine. Le quartier des Grottes/Genève. Genève : MétisPresses. p. 259-283.

Pecqueux, Anthony. 2012. Politiques des ambiances. Que faire du fond inextricablement politique des ambiances. In : Thibaud, Jean-Paul \& Siret, Daniel (eds.). 2012. Ambiances en actes : actes du $2^{\text {nd }}$ congrès international sur les ambiances. Grenoble : RIA, ENSAG. p. 145-150.

Pouchepadass, Jacques. 2000. Les Subaltern Studies ou la critique postcoloniale de la modernité. L'Homme, n’ 156, p. 161-186.

Rancière, Jacques. 2000. Le partage du sensible. Esthétique et politique. Paris : La Fabrique Éditions. Roux, Michel. 2002. Inventer un nouvel art d'habiter. Le ré-enchantement de l'espace. Paris : L'Harmatan.

Saïd, Noha. 2014. Vers une écologie sensible des rues du Caire : le palimpseste des ambiances d'une ville en transition. Thèse de doctorat en architecture et aménagement, Université de Grenoble, École doctorale «science de l'homme, du politique et du territoire, Laboratoire Cresson.

Schmitz, Hermann. 2014. Atmosphären. Munich : Karl Alber Verlag.

Schmitz, Hermann. 2018. Les sentiments comme atmosphères. Communications : Exercices d'ambiance. $\mathrm{n}^{\circ} 102$, p. 51-66. [en ligne] Disponible sur : https://www.persee.fr/doc/ comm_0588-8018_2018_num_102_1_2889 (consulté en avril 2019)

Sennett, Richard. 2002. La chair et la pierre : le corps et la ville dans la civilisation occidentale. Paris : Éd. de la Passion.

Simonnot Nathalie, Balaÿ Olivier \& Frioux Stéphane. 2016. L'ambiance et l'histoire de l'architecture : l'expérience et l'imaginaire sensibles de l'environnement construit. Journal Ambiances, [en ligne] Disponible sur : https://doi.org/10.4000/ambiances.742 (consulté en novembre 2021)

Simpson, Paul. 2014. Spaces of Affect. In : Adams, Paul et al (eds). Ashgate Research Companion on Geographies of Media. Aldershot: Ashgate. p. 329-345.

Soja, Edward. 2010. Seeking Spatial Justice. Minneapolis : University of Minnesota Press. 
Sumartojo, Shanti \& Pink, Sarah (eds). 2019. Atmospheres and the experiential world. Theory and methods. Londres - New York : Routledge.

Thibaud, Jean-Paul. 2013. Ambiances urbaines, écologie sensible. In : Euzen, Agathe \& Eymard, Laurence \& Gall, Françoise. Le développement durable à découvert. Paris : CNRS. p. 120-121.

Thibaud, Jean-Paul. 2012. Petite archéologie de la notion d'ambiance. Communications. $\mathrm{n}^{\circ} 90$ (1), p. 155-174. [en ligne] Disponible sur : https://doi.org/10.3917/commu.090.0155 (consulté en mai 2013)

Thibaud, Jean-Paul. 2002. L'horizon des ambiances urbaines. Communications. $\mathrm{n}^{\circ}$ 73, p. 185-201. [en ligne] Disponible sur : https://doi.org/10.3406/comm.2002.2119 (consulté en avril 2003)

Thomas, Rachel. 2020. Les mises en ambiance de la ville apaisée. Paradoxes et risques d'une aseptisation programmée. Géo-Regards. Varia, n 13, p. 179-194.

Thomas, Rachel. 2018. Une critique sensible de l'urbain. HDR en Sciences Humaines et Aménagement. Grenoble : Université Grenoble Alpes, CRESSON.

Thomas, Rachel et al. 2014. MUSE : les énigmes sensibles des mobilités urbaines contemporaines. Grenoble : CRESSON. ANR 10-ESVS-0013.

Thomas, Rachel (dir.) ; Balez Suzel ; Bérubé, Gabriel \& Bonnet Aurore. 2010. L'aseptisation des ambiances piétonnes au XXI siècle. Entre passivité et plasticité des corps en marche. Grenoble : CRESSON. Paris : PIRVE CNRS MEEDDM.

Thomas, Rachel. 2005. Les trajectoires de l'accessibilité. Bernin : À la Croisée.

Winkin, Yves \& Lavadinho, Sonia. 2008. Enchantment, engineering and pedestrian empowerment: the Geneva case. In : Ingold Tim \& Vergunst L. (eds.). Ways of walking. Ethnography and practice on foot. Aldershot : Ashgate. p. 155-167.

\section{NOTES}

1. Par « civilité ", j'entends cet art poli et courtois de vivre en société, dans le respect des règles et des bienséances, dans l'attention continue à agir avec convenance et tact, à participer à ce qu'une certaine harmonie se maintienne.

2. La notion d'atmosphère a son origine dans la phénoménologie allemande. Chez Schmitz (2014, 2018) et chez Böhme (2017a), les atmosphères renvoient à des affects spatialisés : les dispositions affectives des individus ne sont pas liées à leur état psychique, mais bien aux expériences qu'ils font - en tant que corps sentant - des qualités propres à un lieu. Dans le champ de la géographie des affects anglo-saxonne où la notion est aussi développée, les atmosphères émergent de la présence de corps dans un même espace-temps (McCormack, 2008 ; Anderson, 2009 ; Bissel, 2010). Celles-ci ne sont toutefois pas réductibles à la somme de ces individualités. Indéterminées et plus ou moins partagées, elles désignent des qualités affectives singulières d'une situation, d'intensité variable, qui ont le pouvoir de mobiliser un collectif.

3. Böhme (2020) prolonge ici les analyses de Benjamin (1939) sur l'esthétisation de la politique, considérant que cette esthétisation s'étend aujourd'hui, au-delà du fascisme et des états totalitaires, aux états démocratiques où le capitalisme opère à un stade avancé.

4. Des travaux récents défrichent cette question : Balaÿ, Olivier. 2013. L'ambiance comme objet historique. In : Beck Robert, Krampl Ulrike \& Retaillaud-Bajac, Emmanuelle (eds.). Les cinq sens de la ville du Moyen âge à nos jours. Tours, Presses Universitaires François Rabelais, p. 29-51 ; Saïd, Noha. 2014. Vers une écologie sensible des rues du Caire : le palimpseste des ambiances d'une ville en transition. Thèse de doctorat en architecture et aménagement, Université de Grenoble, 
École doctorale "science de l'homme, du politique et du territoire», Laboratoire Cresson; Simonnot Nathalie, Balaÿ Olivier \& Frioux Stéphane. 2016. L'ambiance et l'histoire de l'architecture: l'expérience et l'imaginaire sensibles de l'environnement construit. Journal Ambiances, [en ligne] Disponible sur : https://doi.org/10.4000/ambiances.742

5. Les critical urban studies (Brenner, Marcuse \& Mayer, 2009 ; Harvey, 1973, 2011 ; Imbroscio \& Davies, 2010 ; Soja, 2010) et les sulbaltern urban studies (Guha \& Gayani, 1988; Merle, 2004 ; Pouchepadass, 2000) - outre qu'elles procèdent toutes deux d'une dénonciation de «l'occidentalo-centrisme » des pensées urbaines critiques - procèdent de la sorte en travaillant empiriquement sur les effets du néolibéralisme en termes de justice et de domination, tout en s'intéressant à la généalogie des espaces et des problèmes. Mais ces courants de pensée n'abordent pas la question des ambiances telle que proposée ici.

6. Par exemple, les expériences de marche en situation de handicap visuel et/ou auditif m'aident à saisir l'épaisseur de l'expérience sensible ordinaire en dé-familiarisant les personnes qui y participent.

7. Je remercie ici Daniel Cefaï qui a accompagné cette réflexion et qui m'a orienté vers cette démultiplication des conceptions possibles de la critique depuis le champ des ambiances.

\section{RÉSUMÉS}

Cet article programmatique propose des pistes de travail pour élaborer une critique de l'urbain depuis le champ des ambiances. Si la critique n'est pas absente des travaux de ce champ, elle reste toutefois orientée en direction du champ de la conception auquel il est classiquement reproché de négliger les dimensions sensibles de l'espace. Une critique de l'urbain depuis le champ des ambiances aurait davantage pour enjeu de clarifier les enjeux sociétaux liés à la transformation programmée des ambiances urbaines. Quelle est la part des ambiances dans la mise en place de processus de normalisation des conduites urbaines ordinaires qui peuvent créer de la vulnérabilité, du trouble dans la civilité, voire des formes d'exclusion ? L'argument central du texte consiste à ouvrir le champ des ambiances à des interactions plus étroites avec la question du politique, principalement avec celle des formes de vie sensibles. Il s'agit de se questionner sur la manière de saisir du politique dans les ambiances - depuis des situations concrètes d'enquête et depuis leur généalogie - en décrivant, en questionnant et en appréciant leur transformation et leur poids dans l'épanouissement de manières d'être et d'être affectées plurielles (190 mots)

This prospective article suggests ways of developing a critique of the urban from the field of ambiences. Whilst critique does exist in this field, it is aimed at the field of architecture and design, which is accused of neglecting the sensory dimensions of space. A critique of the urban from the field of ambiences seeks to demonstrate the need to clarify the societal issues related to the planned transformation of urban ambiences. What is the role of ambiences in setting up processes of normalisation of ordinary urban behaviour that can create vulnerability, antisocial behaviour or even forms of exclusion? The central argument of the text is to open up the field of ambiences to closer interactions with the question of political, mainly sensory forms of life. It is a question of grasping the political in ambiences from concrete situations of investigation and from their genealogy, by describing, questioning and appreciating their transformation and their role in the development of plural ways of being, and of being affected. 
INDEX

Keywords : ambience(s), sensory forms of life, critique, political, urban behaviors

Mots-clés : ambiance(s), formes de vie sensibles, critique, conduites urbaines, politique

\section{AUTEUR}

\section{RACHEL THOMAS}

Rachel THOMAS (rachel.thomas@grenoble.archi.fr) est directrice de recherche au CNRS et membre de l'équipe CRESSON du laboratoire Ambiances, Architectures, Urbanités. Elle est habilitée à diriger des recherches en sciences humaines et en aménagement. Son programme de recherche actuel vise à ouvrir le questionnement sur les ambiances urbaines à leurs dimensions critique et politique à partir d'une focalisation sur les enjeux sociétaux de leurs transformations. Elle coordonne, au sein du laboratoire, le groupe de recherche « Ambiances urbaines, critique, politique » : https://aau.archi.fr/groupes-de-recherche/ambiances-urbaines-critique-politique/ 